\title{
Minimum Variance Unbiased Estimation in the Gompertz Distribution under Progressive Type II Censored Data with Binomial Removals
}

\author{
Ashok Shanubhogue and N. R. Jain \\ Department of Statistics, Sardar Patel University, Vallabh Vidyanagar 388120, India \\ Correspondence should be addressed to N. R. Jain; jain_nirmal@sify.com
}

Received 30 October 2012; Accepted 19 November 2012

Academic Editors: B. L. Granovsky and X. Lu

Copyright (c) 2013 A. Shanubhogue and N. R. Jain. This is an open access article distributed under the Creative Commons Attribution License, which permits unrestricted use, distribution, and reproduction in any medium, provided the original work is properly cited.

\begin{abstract}
This paper deals with the problem of uniformly minimum variance unbiased estimation for the parameter of the Gompertz distribution based on progressively Type II censored data with binomial removals. We have obtained the uniformly minimum variance unbiased estimator (UMVUE) for powers of the shape parameter and its functions. The UMVUE of the variance of these estimators is also given. The UMVUE of (i) pdf, (ii) cdf, (iii) reliability function, and (iv) hazard function of the Gompertz distribution is derived. Further, an exact $(1-\alpha) 100 \%$ confidence interval for the $p$ th quantile is obtained. The UMVUE of pdf is utilized to obtain the UMVUE of $P(X<Y)$. An illustrative numerical example is presented.
\end{abstract}

\section{Introduction}

A Type II censored sample is one for which only $m$ smallest observations in a sample of $n$ items are observed. A generalization of Type II censoring is a progressive Type II censoring. Under this scheme, nunits of the same kind are placed on test at time zero, and $m$ failures are observed. When the first failure is observed, a number $r_{1}$ of surviving units are randomly withdrawn from the test; at the second failure time, $r_{2}$ surviving units are selected randomly and taken out of the experiment, and so on. At the time of $m$ th failure, the remaining $r_{m}=n-r_{1}-r_{2}-\cdots-r_{m-1}-m$ units are removed. Balakrishnan et al. [1] indicated that such scheme can arise in clinical trials where the drop out of patients may be caused by migration or by lack of interest. In such situations, the progressive censoring scheme with random removals is required. Many authors have discussed inference under progressive Type II censored samples using different life distributions including El-Din and Shafay [2], Kim et al. [3] Kim and Han [4], Ali Mousa and Jaheen [5], and Pérez-González and Fernández [6]. For a detailed discussion of progressive censoring, we refer to Balakrishnan and Aggarwala [7] and Balakrishnan [8]. Note that if $r_{1}=$ $r_{2}=\cdots=r_{m-1}=0$, this scheme reduces to the Type II censoring scheme. Also note that if $r_{1}=r_{2}=\cdots=r_{m}=0$, so that $m=n$, the progressively Type II censoring scheme reduces to the case of no censoring, that is, the case of a complete sample. In this paper, we use progressively Type II censoring scheme with binomial removals where the number of units removed at each failure time follows a binomial distribution.

The Gompertz distribution was first introduced by Gompertz [9] to describe human mortality and establish actuarial tables. Since then, many investigators have used the Gompertz distribution or some related forms of it in a variety of studies. There are many forms of the Gompertz distribution in the literature.

The Gompertz distribution is applied in actuarial science, reliability and life testing studies, and epidemiological and biomedical studies. Several such situations have been discussed by Ananda et al. [10], Walker and Adham [11], Jaheen [12], and many others. For a review of literature on estimating parameters of the Gompertz distribution, one may refer to 
Gordon [13], Chen [14], Wu et al. [15], Garg et al. [16], Ismail [17], Al-Khedhairi and El-Gohary [18], and many others.

Inference for The Gompertz distribution based on progressively Type II censored data is discussed by many authors. Wu et al. [19] obtained the maximum likelihood estimators of the two-parameter Gompertz distribution under progressive Type II censoring with binomial removals. They had also given the expected test time to complete the censoring test. Wu et al. [20] discussed the problem of interval estimation for the two-parameter Gompertz distribution under progressive Type II censored data. Many authors have studied the problem of estimation of $P=P(X<Y)$ for various distributions. This model involves two independent random variables $X$ and $Y$. The term $P=P(X<Y)$ is the reliability of a system of strength $Y$ is subjected to a stress $X$. The system fails if the applied stress exceeds its strength. An extensive review of this topic is given in Kotz.et al. [21]. Saraçoğlu and kaya [22] obtained the maximum likelihood estimate of stress strength reliability for the Gompertz distribution. Saraçoğlu et al. [23] have obtained maximum likelihood estimate and UMVUE of stress strength reliability for the Gompertz distribution when $X$ and $Y$ are independent but not identically random variables belonging to the Gompertz distribution when complete sample is available.

In this paper, we discuss the problem of UMVUE for shape parameter of the Gompertz distribution based on progressively Type II censored data with binomial removals. In Section 2, the conditional likelihood function is given. In Section 3, the UMVUE of parameter of $\theta$ and its functions are derived. Also, the UMVUE of the (i) pdf, (ii) cdf, (iii) reliability function (iv) hazard function are obtained. In Section 4 . the UMVUE of $P(X<Y)$ is obtained by using the UMVUE of p.d.f. In Section 5 an exact $(1-\alpha) 100 \%$ confidence interval for $p$ th quantile is obtained. An illustrative numerical example is presented.

\section{The Model}

Let the failure time distribution be the Gompertz with probability density function,

$$
f(x)= \begin{cases}\theta e^{c x} \exp \left\{-\frac{\theta}{c}\left(e^{c x}-1\right)\right\} & \theta>0, c>0, x>0 \\ 0 & \text { otherwise }\end{cases}
$$

where $\theta$ and $c$ are the parameters. We assume that $c$ is known.

The cumulative distribution function is given by

$$
F(x)=1-\exp \left\{-\frac{\theta}{c}\left(e^{c x}-1\right)\right\}, \quad x>0 .
$$

The survival function is given by

$$
S(x)=\exp \left\{-\frac{\theta}{c}\left(e^{c x}-1\right)\right\}, \quad x>0 .
$$

The density given in (1) can be written as

$$
f(x)=\frac{a(x)[h(\theta)]^{d(x)}}{[g(\theta)]},
$$

where

$$
\begin{aligned}
& a(x)=e^{c x}, \quad h(\theta)=\exp (-\theta), \\
& d(x)=\frac{1}{c}\left[e^{c x}-1\right], \quad g(\theta)=\frac{1}{\theta},
\end{aligned}
$$

such that $a(x)>0, \quad g(\theta)=\int_{x>0} a(x)[h(\theta)]^{d(x)} d x$.

Let $\left(X_{1}, R_{1}\right),\left(X_{2}, R_{2}\right), \ldots,\left(X_{m}, R_{m}\right)$ denote a progressively Type II censored sample, where $X_{i}=X_{i: m: n}$, for $i=$ $1,2, \ldots m$, and $X_{1}<X_{2}<\cdots<X_{m}$. The conditional likelihood function can be written as, see Cohen [24],

$$
L(\theta ; \underline{x} / \underline{R}=\underline{r})=A \prod_{i=1}^{m} f\left(x_{i}\right) \cdot\left[s\left(x_{i}\right)\right]^{r_{i}},
$$

where $A=n\left(n-r_{1}-1\right)\left(n-r_{1}-r_{2}-2\right) \cdots\left(n-r_{1}-r_{2}-\right.$ $\left.\cdots r_{m-1}-m+1\right)$ and $0 \leq r_{i} \leq\left(n-m-r_{1}-r_{2}-\cdots r_{i-1}\right)$ for $i=1,2, \ldots m-1$.

Substituting (1) and (3) in (6) we get

$$
\begin{aligned}
& L(\theta ; \underline{x} / \underline{R}=\underline{r}) \\
& \quad=A \theta^{m} e^{c \sum_{i=1}^{m} x_{i}} \exp \left\{-\frac{\theta}{c}\left[\sum_{i=1}^{m}\left(1+r_{i}\right) e^{c x_{i}}-n\right]\right\} .
\end{aligned}
$$

Suppose that an individual unit being removed from the life test is independent of others but with the same probability $p$. Then the number of units removed at each failure time follows a binomial distribution, and, following Wu et al. [19], the joint probability mass function of $r_{1}, r_{2}, \ldots r_{m-1}$ is given by

$$
P(\underline{R}=\underline{r})=\frac{(n-m) ! p^{\sum_{i=1}^{m-1} r_{i}}(1-p)^{(m-1)(n-m)-\sum_{i=1}^{m-1}(m-i) r_{i}}}{\left(n-m-\sum_{i=1}^{m-1} r_{i}\right) ! \prod_{i=1}^{m-1} r_{i} !}
$$

that is,

$$
P(\underline{R}=\underline{r})=\frac{(n-m) !\left[p /(1-p)^{m}\right]^{\sum_{i=1}^{m-1} r_{i}}(1-p)^{\sum_{i=1}^{m-1} i r_{i}}}{\prod_{i=1}^{m} r_{i} !\left[(1-p)^{-(n-m)}\right]^{(m-1)}} .
$$

The unconditional likelihood function is

$$
L(\theta, p ; \underline{x}, \underline{r})=L(\theta ; \underline{x} / \underline{R}=\underline{r}) P(\underline{R}=\underline{r}) .
$$

Using (7) and (9) in (10) we can write the full likelihood function as

$$
\begin{aligned}
L(\theta, \underline{p} ; \underline{x}, \underline{r})= & A \theta^{m} e^{c \sum_{i=1}^{m} x_{i}} \exp \left\{-\frac{\theta}{c}\left[\sum_{i=1}^{m}\left(1+r_{i}\right) e^{c x_{i}}-n\right]\right\} \\
& \times \frac{(n-m) !\left[p /(1-p)^{m}\right]^{\sum_{i=1}^{m-1} r_{i}}(1-p)^{\sum_{i=1}^{m-1} i r_{i}}}{\prod_{i=1}^{m} r_{i} !\left[(1-p)^{-(n-m)}\right]^{(m-1)}} .
\end{aligned}
$$




\section{Unbiased Estimation}

Let

$$
Y_{i}=\frac{1}{c}\left[e^{c X_{i}}-1\right], \quad i=1,2, \ldots, m
$$

then $Y_{i}$ have exponential distribution with mean $1 / \theta$. We can show that $Y_{1}<Y_{2}<\cdots<Y_{m}$ is a progressive Type II censored sample from an exponential distribution with mean $1 / \theta$. Let us consider the following transformation:

$$
\begin{aligned}
& Z_{1}=n Y_{1}, \\
& Z_{i}=\left(n-i+1-r_{1}-r_{2}-\cdots-r_{i-1}\right)\left(Y_{i}-Y_{i-1}\right), \\
& \quad i=2,3, \ldots, m .
\end{aligned}
$$

In order to derive the distribution of $Z_{i}, i=1,2, \ldots, m$ consider the inverse transformation $Y_{1}=Z_{1} / n$ and $Y_{i}=$ $\sum_{i=2}^{m}\left(Z_{i} /\left(n-r_{i-1}-i+1\right)\right), i=2,3, \ldots m$. The variables $Z_{1}, Z_{2}, \ldots, Z_{m}$ defined in (13) are all independent and identically distributed with exponential distribution with mean $1 / \theta$, see Thomas and Wilson [25]. The joint density of $Z_{1}, Z_{2}, \ldots, Z_{m}$, is

$$
f(\underline{z}, \theta / \underline{R}=\underline{r})=\theta^{m} \exp \left\{-\theta \sum_{i=1}^{m} z_{i}\right\} .
$$

It can be seen that

$$
\sum_{i=1}^{m} Z_{i}=\sum_{i=1}^{m}\left(1+r_{i}\right) Y_{i}
$$

Using (12) in (15), we have

$$
\sum_{i=1}^{m} Z_{i}=\frac{1}{c} \sum_{i=1}^{m}\left(1+r_{i}\right)\left[e^{c X_{i}}-1\right]
$$

Let

$$
\begin{aligned}
T & =\sum_{i=1}^{m} Z_{i} . \\
& =\frac{1}{c}\left[\sum_{i=1}^{m}\left(1+r_{i}\right) e^{c X_{i}}-n\right] .
\end{aligned}
$$

Since (14) is a member of exponential family of distributions, $T$ is a complete sufficient statistic for $\theta$. The distribution of $T$ is gamma with parameters $\theta$ and $m$, which is again a member of exponential family of distributions. The pdf of $T$ is given by

$$
f(t, \theta)=\frac{B(t, m)[h(\theta)]^{t}}{[g(\theta)]^{m}}
$$

where

$$
B(t, m)=\frac{t^{m-1}}{\mid \bar{m}}, \quad h(\theta)=\exp (-\theta), \quad g(\theta)=\frac{1}{\theta} .
$$

Jani and Dave [26] have studied the problem of minimum variance unbiased estimation in a class of exponential family of distributions. They have shown that if $X_{1}, X_{2}, \ldots X_{n}$, be a random sample from density of the type given in (4) and the p.d.f. of its complete sufficient statistics can be written as the one given in (18), then the UMVUE of $[h(\theta)]^{k}$ is given by

$$
H_{k, n}=\frac{B(t-k, n)}{B(t, n)}, \quad t>k
$$

and the UMVUE of $[g(\theta)]^{k}$ is

$$
G_{k, n}=\frac{B(t, n+k)}{B(t, n)} .
$$

Following the results derived in Jani and Dave [26], we get the UMVUE of some important parametric functions as given below.

(i) Using (20), the UMVUE of $\exp [-\theta k]$ is

$$
\begin{aligned}
H_{k, m}=\left[1-\frac{k}{(1 / c)\left[\sum_{i=1}^{m}\left(1+r_{i}\right) e^{c x_{i}}-n\right]}\right]^{m-1}, \\
\frac{1}{c}\left[\sum_{i=1}^{m}\left(1+r_{i}\right) e^{c x_{i}}-n\right]>k .
\end{aligned}
$$

(ii) Using (22), the UMVUE of the variance of $H_{k, m}$, is given by,

$$
\begin{aligned}
\widetilde{\operatorname{Var}}\left[H_{k, m}\right]= & {\left[1-\frac{k}{(1 / c)\left[\sum_{i=1}^{m}\left(1+r_{i}\right) e^{c x_{i}}-n\right]}\right]^{2 m-2} } \\
& -\left[1-\frac{2 k}{(1 / c)\left[\sum_{i=1}^{m}\left(1+r_{i}\right) e^{c x_{i}}-n\right]}\right]^{m-1} .
\end{aligned}
$$

(iii) Using (21), the UMVUE of $(1 / \theta)^{k}$, is given by

$$
G_{k, m}=\frac{\mid \bar{m}}{\mid \overline{m+k}}\left[\frac{1}{c}\left[\sum_{i=1}^{m}\left(1+r_{i}\right) e^{c x_{i}}-n\right]\right]^{k} .
$$

(iv) Using (24), the UMVUE of the variance of $G_{k, m}$ is given by

$$
\begin{aligned}
\widetilde{V} & \operatorname{ar}\left[G_{k, m}\right] \\
& =\left[\frac{1}{c}\left[\sum_{i=1}^{m}\left(1+r_{i}\right) e^{c x_{i}}-n\right]\right]^{2 k}\left[\left(\frac{\mid \bar{m}}{\sqrt{m+k}}\right)^{2}-\left(\frac{\mid \bar{m}}{\overline{m+2 k}}\right)\right] .
\end{aligned}
$$

(v) The UMVUE of density $f(x)$ given in (1), for fixed $x$, is given by,

$$
\begin{array}{r}
\phi_{x, m}=\left(\frac{(m-1) e^{c x}}{t}\right)\left(1-\frac{\left[e^{c x}-1\right]}{c t}\right)^{m-2}, \\
\frac{1}{c}\left[e^{c x}-1\right]<t<\infty, m>2,
\end{array}
$$


where

$$
t=\frac{1}{c}\left[\sum_{i=1}^{m}\left(1+r_{i}\right) e^{c x_{i}}-n\right] .
$$

(vi) The UMVUE of variance of $\phi_{x, m}, m>2$ is given by

$$
\begin{aligned}
& \text { Var }\left[\phi_{x, m}\right] \\
& =\left\{\begin{array}{l}
\left(\frac{(m-1) e^{c x}}{t}\right)^{2}\left[1-\frac{\left[e^{c x}-1\right]}{c t}\right]^{2 m-4} \\
-\left(\frac{(m-1) e^{c x}}{t}\right) \times\left[1-\frac{\left[e^{c x}-1\right]}{c t}\right]^{m-2} \\
\times\left(\frac{c(m-2) e^{c x}}{c t-\left[e^{c x}-1\right]}\right)\left[1-\frac{\left[e^{c x}-1\right]}{\left[c t-\left[e^{c x}-1\right]\right]}\right]^{m-3}, \\
t>\frac{2}{c}\left[e^{c x}-1\right] \\
\left(\frac{(m-1) e^{c x}}{t}\right)^{2} \times\left[1-\frac{\left[e^{c x}-1\right]}{c t}\right]^{2 m-4}, \\
\frac{1}{c}\left[e^{c x}-1\right]<t \leq \frac{2}{c}\left[e^{c x}-1\right]
\end{array}\right.
\end{aligned}
$$

where $t$ is given by (27).

(vii) Considering $x$ as fixed, the UMVUE of reliability function $R(x)=P(X>x), x \geq 0$ is obtained as follows. Since $R(x)=[h(\theta)]^{\left[e^{c x}-1\right] / c}$, where $h(\theta)$ is given in (5) and using (22) with $k=(1 / c)\left[e^{c x}-1\right]$, the UMVUE $\widetilde{R}(x)$ of $R(x)$ is given by

$$
\widetilde{R}(x)=\left[1-\frac{\left[e^{c x}-1\right]}{\left[\sum_{i=1}^{m}\left(1+r_{i}\right) e^{c x_{i}}-n\right]}\right]^{m-1} .
$$
given by

(viii) Using (29), the UMVUE of the variance of $\widetilde{R}(x)$ is

$$
\begin{aligned}
& \text { Var }[\widetilde{R}(x)] \\
& =\left\{\begin{array}{l}
{\left[1-\frac{\left[e^{c x}-1\right]}{c t}\right]^{2 m-2}-\left[1-\frac{2\left[e^{c x}-1\right]}{c t}\right]^{m-1}} \\
0<x<\frac{1}{2 c} \log [c t+2] \\
{\left[1-\frac{\left[e^{c x}-1\right]}{c t}\right]^{2 m-2}} \\
\frac{1}{2 c} \log [c t+2]<x<\frac{1}{2 c} \log [c t+1] \\
0 \quad \text { otherwise. }
\end{array}\right.
\end{aligned}
$$

(ix) The UMVUE of cumulative distribution function given in (2) is

$\widetilde{F}(x)$

$$
=\left\{\begin{array}{cc}
0, & x<0 \\
1-\left(1-\frac{\left[e^{c x}-1\right]}{\left[\sum_{i=1}^{m}\left(1+r_{i}\right) e^{c x_{i}}-n\right]}\right)^{m-1}, \\
& 0<x<\frac{1}{c} \log \left[\sum_{i=1}^{m}\left(1+r_{i}\right) e^{c x_{i}}-n+1\right] \\
1 & \text { otherwise. }
\end{array}\right.
$$

\section{Special Cases}

(a) Substituting $k=1$ in (22), we get the UMVUE of $\exp [-\theta]$ as,

$$
\begin{array}{r}
H_{1, m}=\left[1-\frac{1}{(1 / c)\left[\sum_{i=1}^{m}\left(1+r_{i}\right) e^{c x_{i}}-n\right]}\right]^{m-1}, \\
\frac{1}{c}\left[\sum_{i=1}^{m}\left(1+r_{i}\right) e^{c x_{i}}-n\right]>1 .
\end{array}
$$

(b) Substituting $k=-1$ in (22), we get UMVUE of $\exp [\theta]$ as

$$
\begin{array}{r}
H_{-1, m}=\left[1+\frac{1}{(1 / c)\left[\sum_{i=1}^{m}\left(1+r_{i}\right) e^{c x_{i}}-n\right]}\right]^{m-1}, \\
\frac{1}{c}\left[\sum_{i=1}^{m}\left(1+r_{i}\right) e^{c x_{i}}-n\right]>0 .
\end{array}
$$

(c) Substituting $k=1$ in (24), we get the UMVUE of $(1 / \theta)$ as

$$
G_{1, m}=\frac{\left[\sum_{i=1}^{m}\left(1+r_{i}\right) e^{c x_{i}}-n\right]}{c m} .
$$

(d) Substituting $k=-1$ in (24), we get the UMVUE of $\theta$ as

$$
G_{-1, m}=\frac{c(m-1)}{\left[\sum_{i=1}^{m}\left(1+r_{i}\right) e^{c x_{i}}-n\right]} .
$$

(e) The hazard function for the Gompertz distribution is $h(x)=\theta e^{c x}$. Using (35), the UMVUE of hazard function, for fixed $x$, can be given as

$$
\widetilde{h}(x)=\frac{c(m-1) e^{c x}}{\left[\sum_{i=1}^{m}\left(1+r_{i}\right) e^{c x_{i}}-n\right]} .
$$

Shanubhogue and Jain [27] have studied the problem of minimum variance unbiased estimation in exponential distribution under progressive Type II censored data with binomial removals. They have given the UMVUE for parameter $p$ and various functions of $p$. Since the joint density $P(\underline{R}=\underline{r})$ given in (9) is independent of $\theta$, one gets the same estimators of $p$, and its various functions as given in Shanubhogue and Jain [27]. 


\section{UMVU Estimator of $P(X<Y)$}

In the following theorem, we derive the UMVUE of $P(X<Y)$. Let $m_{1}$ units (out of $n_{1}$ ) on $X$ and $m_{2}$ units (out of $n_{2}$ ) on $Y$ are recorded which follow the Gompertz distributions, given in (1) with parameters $\theta_{1}$ and $\theta_{2}$, respectively. Let $r_{1}, r_{2}, \ldots r_{m_{1}}$ and $s_{1}, s_{2}, \ldots s_{m_{2}}$ be corresponding removals. We denote

$$
\begin{aligned}
& t_{1}=\frac{1}{c}\left[\sum_{i=1}^{m_{1}}\left(1+r_{i}\right) e^{c x_{i}}-n_{1}\right], \\
& t_{2}=\frac{1}{c}\left[\sum_{i=1}^{m_{2}}\left(1+s_{i}\right) e^{c y_{i}}-n_{2}\right] .
\end{aligned}
$$

Theorem 1. Under progressive Type II censored data, the UMVU estimator of $P=P(X<Y)$ for the density given in (1) is given by

$$
\widetilde{P}= \begin{cases}\sum_{j=0}^{m_{2}-1}(-1)^{j} \frac{\left(m_{1}-1\right) !\left(m_{2}-1\right) !}{\left(m_{1}+j-1\right) !\left(m_{2}-j-1\right) !}\left(\frac{t_{1}}{t_{2}}\right)^{j} & t_{1}<t_{2} \\ 1-\sum_{i=0}^{m_{1}-1}(-1)^{i} \frac{\left(m_{1}-1\right) !\left(m_{2}-1\right) !}{\left(m_{1}-i-1\right) !\left(m_{2}+i-1\right) !}\left(\frac{t_{2}}{t_{1}}\right)^{i} & t_{1} \geq t_{2},\end{cases}
$$

where $t_{1}$ and $t_{2}$ are given by (37) and (38), respectively.

Proof. We have

$$
\widetilde{P}=\iint_{G} \phi_{x, m_{1}} \phi_{y, m_{2}} d x d y
$$

where

$$
\begin{aligned}
& G=\left\{(x, y): 0<x<\frac{1}{c} \log \left[1+c t_{1}\right],\right. \\
& \left.\quad 0<y<\frac{1}{c} \log \left[1+c t_{2}\right], x<y\right\} .
\end{aligned}
$$

Using (26) in (40) and let $t_{1}<t_{2}$, we have

$$
\begin{aligned}
\widetilde{P}=\int_{0}^{(1 / c) \log \left[1+c t_{1}\right]} \int_{x}^{(1 / c) \log \left[1+c t_{2}\right]}\left[\frac{e^{c x}\left(m_{1}-1\right)}{t_{1}}\right] \\
\times\left[1-\frac{\left(e^{c x}-1\right)}{c t_{1}}\right]^{m_{1}-2}\left[\frac{e^{c y}\left(m_{2}-1\right)}{t_{2}}\right] \\
\times\left[1-\frac{\left(e^{c y}-1\right)}{c t_{2}}\right]^{m_{2}-2} d y d x .
\end{aligned}
$$

Now

$$
\begin{gathered}
\int_{x}^{(1 / c) \log \left[1+c t_{2}\right]}\left[\frac{e^{c y}\left(m_{2}-1\right)}{t_{2}}\right]\left[1-\frac{\left(e^{c y}-1\right)}{c t_{2}}\right]^{m_{2}-2} d y \\
=\left[1-\frac{\left(e^{c x}-1\right)}{c t_{2}}\right]^{m_{2}-1}
\end{gathered}
$$

After substituting (43) into (42), we get

$$
\begin{gathered}
=\int_{0}^{(1 / c) \log \left[1+c t_{1}\right]}\left[\frac{e^{c x}\left(m_{1}-1\right)}{t_{1}}\right]\left[1-\frac{\left(e^{c x}-1\right)}{c t_{1}}\right]^{m_{1}-2} \\
\quad \times\left[1-\frac{\left(e^{c x}-1\right)}{c t_{2}}\right]^{m_{2}-1} d x .
\end{gathered}
$$

Further simplification of (44) and applying the result $(1-w)^{n}=\sum_{j=0}^{n}\left(\begin{array}{c}n \\ j\end{array}\right)(-1)^{j} w^{j}$, we get

$$
=\left(m_{1}-1\right) \sum_{j=0}^{m_{2}-1}\left(\begin{array}{c}
m_{2}-1 \\
j
\end{array}\right)(-1)^{j}\left(\frac{t_{1}}{t_{2}}\right)^{j} \int_{0}^{1} w^{j}(1-w)^{m_{1}-2} d w .
$$

Further simplification of (45) gives

$$
\widetilde{P}=\sum_{j=0}^{m_{2}-1}(-1)^{j} \frac{\left(m_{1}-1\right) !\left(m_{2}-1\right) !}{\left(m_{1}+j-1\right) !\left(m_{2}-j-1\right) !}\left(\frac{t_{1}}{t_{2}}\right)^{j}, \quad t_{1}<t_{2} .
$$

Similarly, we can show that for the case $t_{1}>t_{2}$, the UMVUE of $P(X<Y)$ is

$$
\widetilde{P}=1-\sum_{i=0}^{m_{1}-1}(-1)^{i} \frac{\left(m_{1}-1\right) !\left(m_{2}-1\right) !}{\left(m_{1}-i-1\right) !\left(m_{2}+i-1\right) !}\left(\frac{t_{2}}{t_{1}}\right)^{i} t_{1}>t_{2} .
$$

\section{Exact Confidence Interval for $p$ th Quantile}

Theorem 2. Under progressive Type II censored data an exact $(1-\alpha) 100 \%$ confidence interval for pth quantile, $0<p<1$ is given by,

$$
\left[\frac{1}{c} \log \left\{\frac{2 m\left[e^{c \tilde{\zeta}_{p}}-1\right]}{\chi_{2 m, 1-\alpha / 2}^{2}}+1\right\}, \frac{1}{c} \log \left\{\frac{2 m\left[e^{c \tilde{\zeta}_{p}}-1\right]}{\chi_{2 m, \alpha / 2}^{2}}+1\right\}\right] .
$$

Proof. Using (2) and (35), we have

$$
e^{c \tilde{\xi}_{p}}=1+\left(\frac{t}{m}\right) c \log \left[\frac{1}{(1-p)}\right] .
$$

Hence, $t=m\left[e^{c \tilde{\xi}_{p}}-1\right] / c \log [1 /(1-p)]$. Now, $t$ has gamma distribution with parameters $\theta$ and $m$.

Using the relation $c \log [1 /(1-p)]=\theta\left[e^{c \xi_{p}}-1\right]$, we make the transformation $Q=2 T \theta$ where

$$
Q=\frac{2 m\left[e^{c \tilde{\xi}_{p}}-1\right]}{\left[e^{c \xi_{p}}-1\right]} .
$$

Now, $Q$ has $\chi^{2}$ distribution with $2 m$ degrees of freedom. Thus,

$$
P\left\{\chi_{2 m, \alpha / 2}^{2}<Q<\chi_{2 m, 1-\alpha / 2}^{2}\right\}=1-\alpha .
$$

Using (50) and rearranging (51), we get (48). 
TABle 1: Progressive Type II censored sample with binomial removals generated from the tumor-free time data.

\begin{tabular}{lll}
\hline$i$ & $x_{i}$ & $r_{i}$ \\
\hline 1 & 60 & 1 \\
2 & 63 & 1 \\
3 & 66 & 0 \\
4 & 66 & 0 \\
5 & 66 & 0 \\
6 & 68 & 1 \\
7 & 70 & 1 \\
8 & 77 & 1 \\
9 & 91 & 1 \\
10 & 94 & 0 \\
11 & 98 & 0 \\
12 & 101 & 0 \\
13 & 105 & 0 \\
14 & 108 & 1 \\
15 & 112 & 0 \\
16 & 112 & 1 \\
17 & 126 & 0 \\
18 & 143 & 4 \\
\hline
\end{tabular}

\section{Illustrative Example}

In this section, we illustrate the use of the estimation methods given in this paper.

The following are the numbers of tumor-free days of 30 rats fed with unsaturated diet, see King et al. [28]. The data are

$$
112,68,84,109,153,143,60,70,98,164,63,63,77,91,91,
$$

$66,70,77,63,66,66,94,101,105,108,112,115,126,161,178$.

These data are presented by Lee [29] and studied by Chen [14] and $\mathrm{Wu}$ et al. [20]. Chen [14] and $\mathrm{Wu}$ et al. [20] assumed a Gompertz distribution for tumor-free times. Wu et al. [20] obtained the MLE of $c$ as $\widehat{c}=0.0505$ for progressive Type II censored data. We generate a progressive Type II censored data with binomial removals from these data, assuming $c=$ 0.0505 . The progressive censored sample size is $m=18$. The dropout numbers have been generated using MYSTAT software as follows: $r_{1}$ from $B(12,0.1)$ and $r_{i} / r_{1}, r_{2}, \ldots, r_{i-1}$ have $B\left(12-\sum_{j=1}^{i-1} r_{j}, 0.1\right)$ distribution for $i=2,3, \ldots 17$ and set

$$
r_{18}= \begin{cases}12-\sum_{j=1}^{17} r_{j} & \text { if } 12-\sum_{j=1}^{17} r_{j}>0 \\ 0 & \text { otherwise. }\end{cases}
$$

Using the results given in Section 3, the UMVU estimates of different parametric functions of $\theta$ based on data given in Table 1 are given in Table 2.

An exact 95\% confidence interval for the third quartile, that is, $p=0.75$, is $(123.35,141.87)$.
TABLE 2: The UMVU estimates of different parametric functions of $\theta$ based on data given in Table 1 .

\begin{tabular}{lcc}
\hline $\begin{array}{l}\text { Serial } \\
\text { Number }\end{array}$ & Parametric function & UMVU estimate \\
\hline 1 & $\exp [-\theta]$ & $H_{1,18}=0.999914$ \\
2 & Variance of $H_{1,18}$ & $\widetilde{V} \operatorname{ar}\left[H_{1,18}\right]=4.38788 E-10$ \\
3 & $\exp [\theta]$ & $H_{-1,18}=1.000086378$ \\
4 & Variance of $H_{-1,18}$ & $\widetilde{V} \operatorname{ar}\left[H_{-1,18}\right]=4.38932 E-10$ \\
5 & $1 / \theta$ & $G_{1,18}=10934.27603$ \\
6 & Variance of $G_{1,18}$ & $\widetilde{V} \operatorname{ar}\left[G_{1,18}\right]=6292546.967$ \\
7 & $\theta$ & $G_{-1,18}=8.63747 E-5$ \\
8 & Variance of $G_{-1,18}$ & $\widetilde{V} \operatorname{ar}\left[G_{-1,18}\right]=4.38858 E-10$ \\
9 & Hazard function at $x=95$. & $\widetilde{h}(x)=0.010469216$ \\
10 & Density at $x=95$ & $\phi_{95,18}=0.008617136$ \\
11 & Variance of $\phi_{95,18}$ & $\widetilde{V} \operatorname{ar}\left[\phi_{95,18}\right]=2.80714 E-6$ \\
12 & Reliability at $x=95$ & $\widetilde{R}(x)=0.81314$ \\
13 & Variance of $\widetilde{R}(x)$ at $x=95$ & $\widetilde{V} \operatorname{ar}[\widetilde{R}(x)]=0.001683$ \\
14 & c.d.f at $x=95$ & $\widetilde{F}(x)=0.186862$ \\
\hline
\end{tabular}

\section{Acknowledgment}

The authors wish to thank the referees for valuable comments that led to the improvement of this paper.

\section{References}

[1] N. Balakrishnan, E. Cramer, U. Kamps, and N. Schenk, "Progressive type II censored order statistics from exponential distributions," Statistics, vol. 35, no. 4, pp. 537-556, 2001.

[2] M. M. M. El-Din and A. R. Shafay, "One- and two-sample Bayesian prediction intervals based on progressively Type-II censored data," Statistical Papers. In press.

[3] C. Kim, J. Jung, and Y. Chung, "Bayesian estimation for the exponentiated Weibull model under Type II progressive censoring," Statistical Papers, vol. 52, no. 1, pp. 53-70, 2011.

[4] C. Kim and K. Han, "Estimation of the scale parameter of the half-logistic distribution under progressively type II censored sample," Statistical Papers, vol. 51, no. 2, pp. 375-387, 2010.

[5] M. A. M. Ali Mousa and Z. F. Jaheen, "Bayesian prediction for progressively censored data from the Burr model," Statistical Papers, vol. 43, no. 4, pp. 587-593, 2002.

[6] C. J. Pérez-González and A. J. Fernández, "Accuracy of approximate progressively censored reliability sampling plans for exponential models," Statistical Papers, vol. 50, no. 1, pp. 161-170, 2009.

[7] N. Balakrishnan and R. Aggarwala, Progressive Censoring: Theory, Methods and Applications, Birkhauser, Boston, Mass, USA, 2000.

[8] N. Balakrishnan, "Progressive censoring methodology: an appraisal," Test, vol. 16, no. 2, pp. 211-259, 2007.

[9] B. Gompertz, "On the nature of the function expressive of the law of human mortality and on the new mode of determining the value of life contingencies," Philosophical Transactions of the Royal Society A, vol. 115, pp. 513-580, 1825.

[10] M. M. Ananda, R. J. Dalpatadu, and A. K. Singh, "Adaptive bayes estimators for parameters of the Gompertz survival model," 
Applied Mathematics and Computation, vol. 75, no. 2-3, pp. 167$177,1996$.

[11] S. G. Walker and S. A. Adham, "A multivariate Gompertz-type distribution," Journal of Applied Statistics, vol. 28, pp. 1051-1065, 2001.

[12] Z. F. Jaheen, "A Bayesian analysis of record statistics from the Gompertz model," Applied Mathematics and Computation, vol. 145, no. 2-3, pp. 307-320, 2003.

[13] N. H. Gordon, "Maximum likelihood estimation for mixtures of two Gompertz distribution when censoring occurs," Communications in Statistics-Simulation and Computation, vol. 19, pp. 733-747, 1990.

[14] Z. Chen, "Parameter estimation of the Gompertz population," Biometrical Journal, vol. 39, no. 1, pp. 117-124, 1997.

[15] J.-W. Wu, W.-L. Hung, and C.-H. Tsai, "Estimation of parameters of the Gompertz distribution using the least squares method," Applied Mathematics and Computation, vol. 158, no. 1, pp. 133-147, 2004.

[16] M. L. Garg, B. R. Rao, and C. K. Redmond, "Maximum likelihood estimation of the parameters of the Gompertz survival function," Journal of the Royal Statistical Society C, vol. 19, pp. 152-159, 1970.

[17] A. A. Ismail, "Bayes estimation of Gompertz distribution parameters and acceleration factor under partially accelerated life tests with type-I censoring," Journal of Statistical Computation and Simulation, vol. 80, no. 11, pp. 1253-1264, 2010.

[18] A. Al-Khedhairi and A. El-Gohary, "A new class of bivariate Gompertz distributions and its mixture," International Journal of Mathematical Analysis, vol. 2, pp. 235-253, 2008.

[19] C.-C. Wu, S.-F. Wu, and H.-Y. Chan, "MLE and the estimated expected test time for the two-parameter Gompertz distribution under progressive censoring with binomial removals," Applied Mathematics and Computation, vol. 181, no. 2, pp. 16571670, 2006.

[20] S.-J. Wu, C.-T. Chang, and T.-R. Tsai, "Point and interval estimations for the Gompertz distribution under progressive type-II censoring," Metron, vol. 61, pp. 403-418, 2003.

[21] S. Kotz, Y. Lumelsdii, and M. Pensky, The Stress-Strength Model and Its Generalization, World Scientific, Singapore, 2003.

[22] B. Saraçoğlu and M. F. Kaya, "Maximum likelihood estimation and confidence intervals of system reliability for Gompertz distribution in stress-strength models," Selçuk Journal of Applied Mathematics, vol. 8, no. 2, pp. 25-36, 2007.

[23] B. Saraçoğlu, M. F. Kaya, and A. M. Abd-Elfattah, "Comparison of estimators for stress-strength reliability in the gompertz case," Hacettepe Journal of Mathematics and Statistics, vol.38, no. 3, pp. 339-349, 2009.

[24] A. C. Cohen, "Progressively censored samples in life testing," Technometrics, vol. 5, pp. 327-329, 1963.

[25] D. R. Thomas and W. M. Wilson, "Linear order statistic estimation for the two parameter Weibull and extreme value distributions from Type II progressively censored samples," Technometrics, vol. 14, pp. 679-691, 1972.

[26] P. N. Jani and H. P. Dave, "Minimum variance unbiased estimation in a class of exponential family of distributions and some of its applications," Metron, vol. 48, no. 1-4, pp. 493-507, 1990.

[27] A. Shanubhogue and N. R. Jain, "Minimum variance unbiased estimation in exponential distribution using progressively type II censored data with binomial removals," Advances and Applications in Statistics, vol. 18, pp. 27-39, 2010.
[28] M. M. King, D. M. Bailey, D. D. Gibson, J. V. Pitha, and P. B. McCay, "Incidence and growth of mammary tumors induced by 7,12-dimethylbenz $[\alpha]$ anthracene as related to the dietary content of fat and antioxidant," Journal of the National Cancer Institute, vol. 63, no. 3, pp. 657-663, 1979.

[29] E. T. Lee, Statistical Methods for Survival Data Analysis, Wiley, New York, NY, USA, 2nd edition, 1992. 


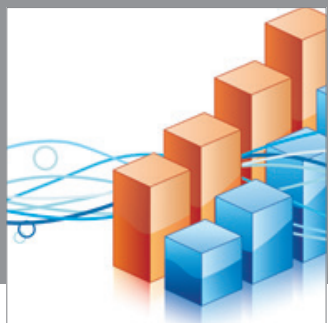

Advances in

Operations Research

mansans

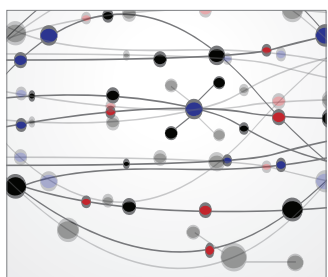

The Scientific World Journal
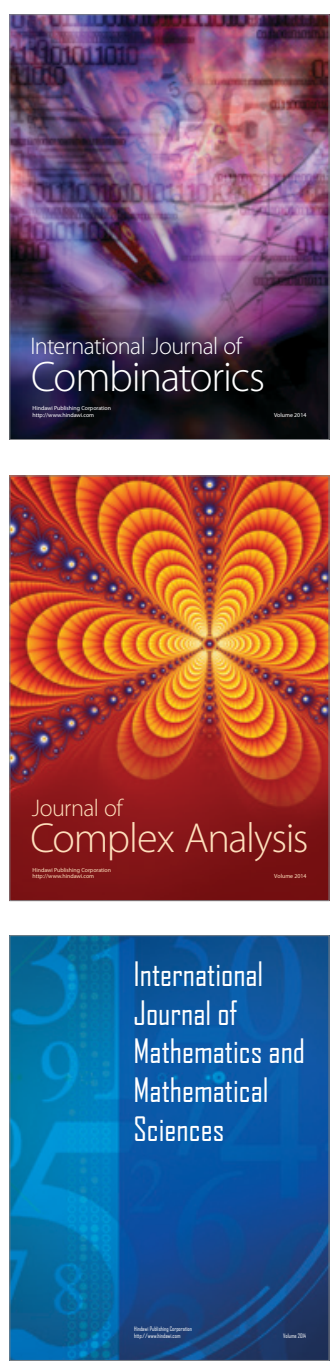
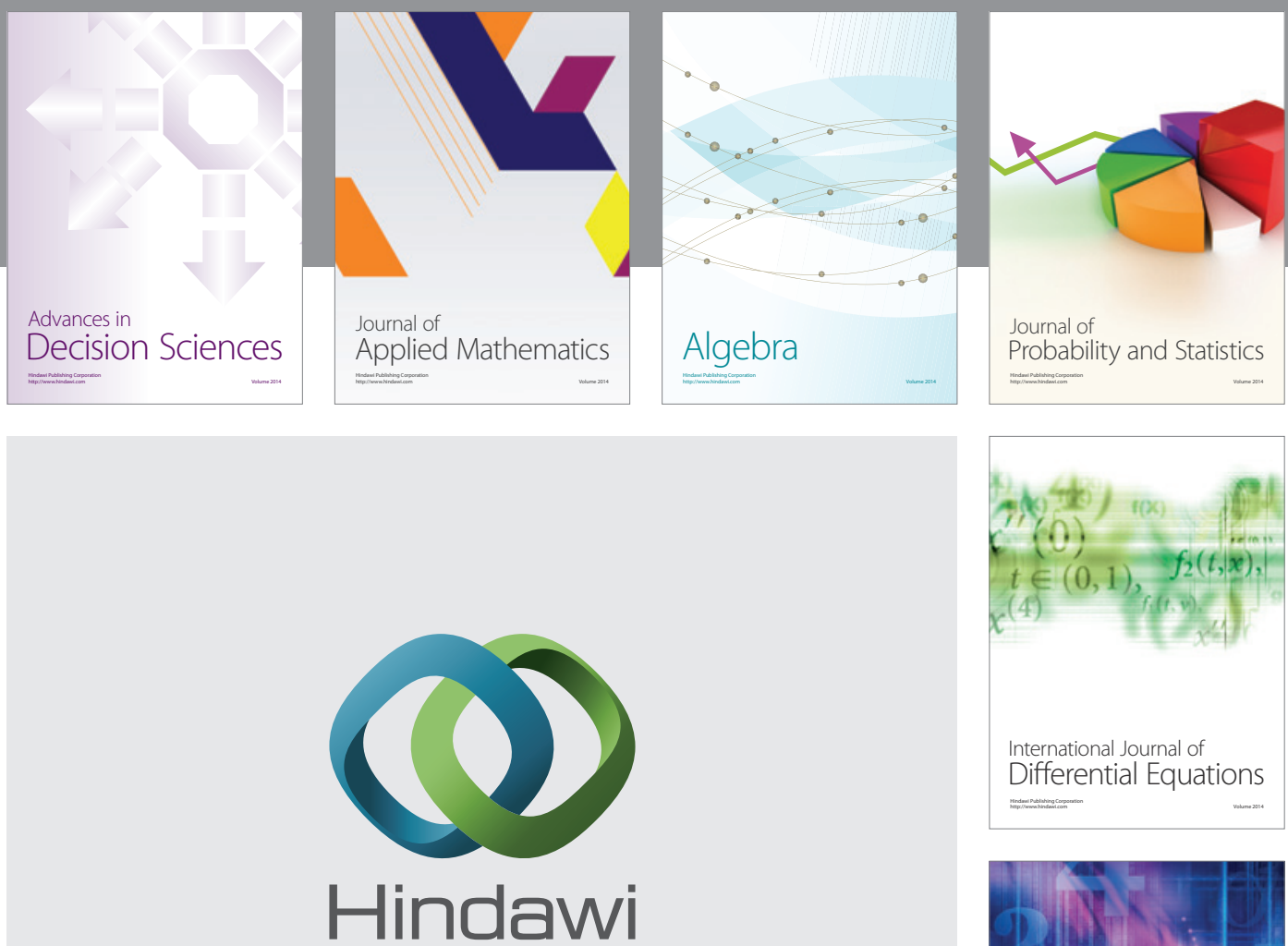

Submit your manuscripts at http://www.hindawi.com
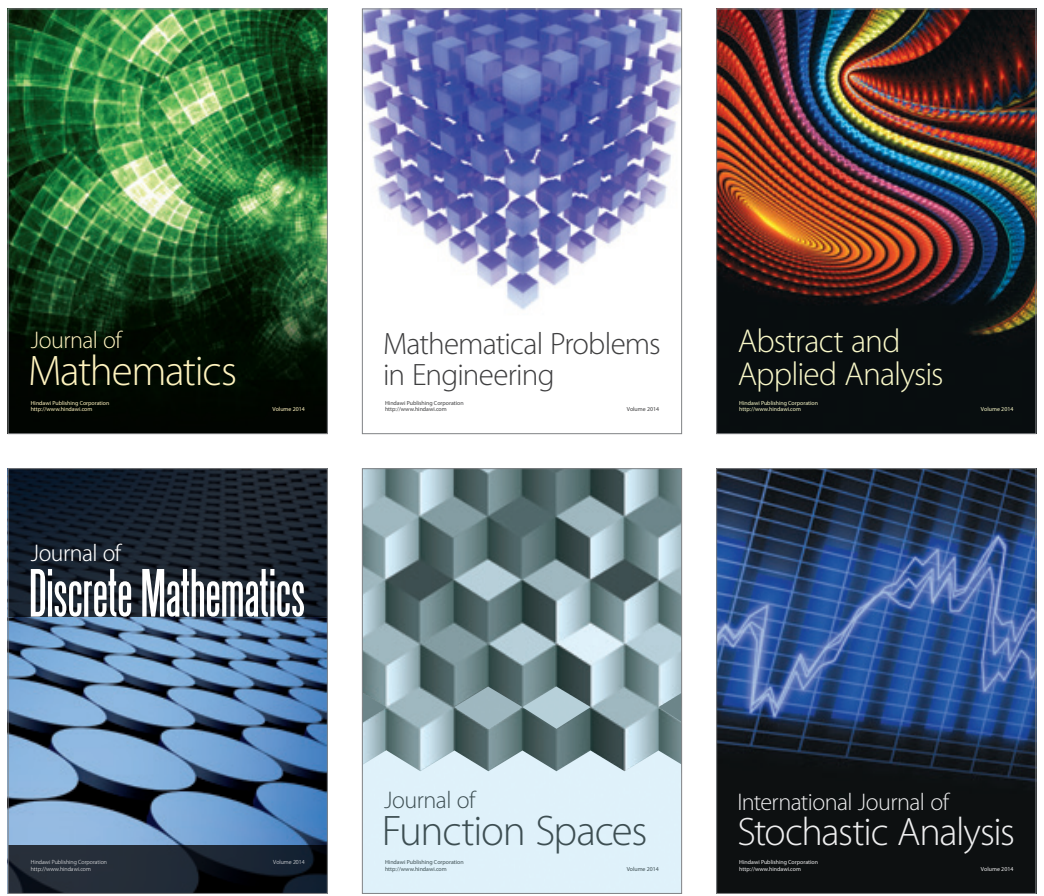

Journal of

Function Spaces

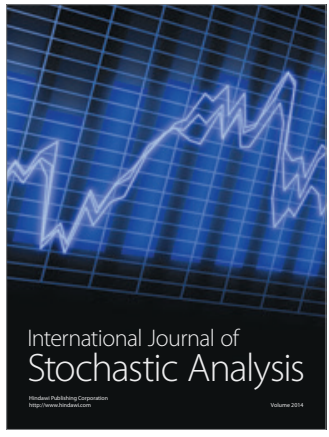

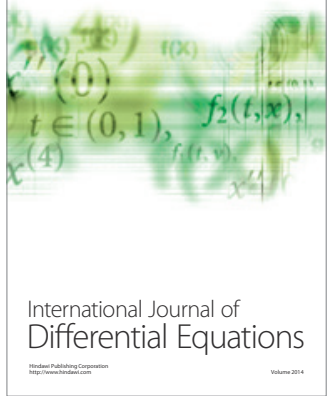
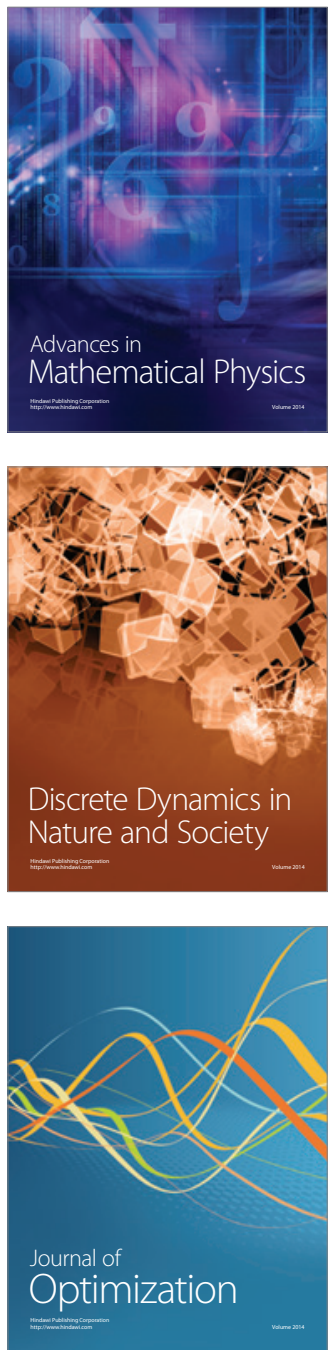